\title{
Dual-contrast echo planar imaging with keyhole: application to dynamic contrast-enhanced perfusion studies
}

\author{
M Zaitsev ${ }^{1,4}$, J D’Arcy², D J Collins², M O Leach², K Zilles ${ }^{1}$ \\ and $\mathbf{N} \mathbf{J}$ Shah $^{1,3}$ \\ ${ }^{1}$ Institut für Medizin, Forschungszentrum Jülich GmbH, 52425 Jülich, Germany \\ ${ }^{2}$ Cancer Research UK, Clinical Magnetic Resonance Research Group, \\ Institute of Cancer Research, The Royal Marsden NHS Trust, Downs Road, Sutton, Surrey SM2 \\ 5PT, UK \\ ${ }^{3}$ Institute of Physics, University of Dortmund, 44221 Dortmund, Germany \\ E-mail: n.j.shah@fz-juelich.de
}

Received 3 February 2005, in final form 1 June 2005

Published 14 September 2005

Online at stacks.iop.org/PMB/50/4491

\begin{abstract}
A new EPI-based method is presented which features optimized sampling of $k$-space enabling the integrated acquisition of two gradient echo images. The first of these images is predominantly $T_{1}$ weighted and the second is $T_{2}^{*}$ weighted. The new method combines echo sharing of sparsely acquired high spatial frequency components with the keyhole technique and half-Fourier image reconstruction. The feasibility of acquiring high spatial and temporal resolution in vivo images for perfusion mapping is demonstrated. In contrast to most current perfusion methods, which acquire the $T_{1^{-}}$and $T_{2}^{*}$-weighted images in separate acquisitions, the need for image co-registration here is obviated since both sets of images are EPI-based and are acquired within the same measurement.
\end{abstract}

\section{Introduction}

MRI provides the means to access a large number of physiological parameters that are of importance in diagnosis and therapy. Many methods have been developed to enable the noninvasive measurement of regional blood volume and flow, tissue permeability and the uptake and washout of the contrast agent. These techniques are based on either endogenous (blood) or exogenous contrast agents. Diagnostic perfusion imaging of the human brain is, beyond doubt, one of the most important and widespread applications of these perfusion techniques.

${ }^{4}$ Present address: Department of Diagnostic Radiology, Medical Physics, University Hospital Freiburg, 79106 Freiburg, Germany. 
The methodology of brain perfusion imaging has recently been reviewed by Barbier et al (2001).

In a typical perfusion experiment, following an intravenous bolus injection of a contrast agent, most commonly gadopentetate dimeglumine (Gd-DTPA), arrival of the bolus in the brain microvasculature may be tracked through the acquisition of a time series of images. Here, both the spatial resolution of the images and the temporal resolution with which they are acquired are critical, as is the contrast induced in the images through the choice of MR sequence parameters. A gradient echo (GE) sequence, typically a spoiled gradient echo (FLASH), is most commonly used to track the influx of contrast agent with a temporal resolution of about $2 \mathrm{~s}$ and an in-plane spatial resolution of 1-2 mm for a single-slice acquisition. Despite the fact that FLASH-based techniques in dynamic contrast-enhanced studies provide valuable functional information, the use of methods based on a single image is considered to be inadequate for the accurate quantification of the required parameters. This results mainly from the combined effects of both $T_{1}$ and $T_{2}^{*}$ relaxation time shortening during bolus passage, which may limit changes in image contrast. On the other hand, as demonstrated recently (Barbier et al 2001, d'Arcy et al 2002), acquiring images with different types of contrasts during the bolus passage delivers new and useful information which can aid accurate quantification of clinically useful parameters such as tissue permeability, cerebral blood volume (CBV), cerebral blood flow (CBF), mean transit time (MTT) and so on. In particular, acquisition of two types of images during the bolus passage, where one is predominantly $T_{1}$ weighted and the other is predominantly $T_{2}^{*}$ weighted, allows both permeability and perfusion to be analysed (Barbier et al 1999, Kim et al 2004).

A majority of the current dual-contrast techniques utilize GE-based sequences to acquire $T_{1}$-weighted images. Differences in the methods arise from the way in which the $T_{2}^{*}$-weighted images are acquired; some use double-echo GE sequences and acquire the second, $T_{2}^{*}$ weighted, image within the same sequence (Vonken et al 2000). Others have acquired $T_{2}$-weighted images using echo planar imaging (EPI) in a separate measurement (Aronen et al 1995). It should be noted that both these approaches present some problems: acquisition of the second echo in FLASH-based techniques considerably reduces temporal resolution, especially when strong $T_{2}^{*}$ weighting is required. $T_{2}^{*}$-weighted EPI images, on the other hand, exhibit considerable co-registration problems because of the inevitable spatial distortions introduced by the method, when compared with FLASH, for example.

Dynamic MRI applications place stringent and conflicting demands on the imaging sequences, requiring both high spatial resolution to be able to resolve anatomical detail and high temporal resolution to enable monitoring of rapid signal changes. Since high-performance gradient systems are an integral part of state-of-the-art clinical machines, EPI has become the method of choice for dynamic imaging applications. Even though single-shot EPI is indeed one of the fastest imaging techniques available, it does suffer from inherent shortcomings which limit its application, such as poor spatial resolution, spatial distortions, susceptibility-induced signal drop-out and a long minimum echo time. Multiple-shot EPI methods circumvent some of these limitations and improve image quality and resolution significantly by compromising temporal resolution. In an effort to combine the high temporal resolution of single-shot EPI with the superior spatial resolution and image quality of multi-shot EPI, echo planar imaging with keyhole, EPIK, was proposed (Shah and Zilles 2003, Zaitsev et al 2001). EPIK merges sparsely acquired higher spatial frequency components with an extensively sampled centre of $k$-space. A sliding window approach is used to fill the missing lines of the $k$-space. Such a scheme effectively shortens the readout time in comparison with single-shot EPI and, consequently, problems associated with low bandwidth in the phase-encode direction become less dominant. In terms of temporal resolution, EPIK is superior to interleaved, multi-shot 
EPI because the latter either does not use sliding window reconstruction or, when a sliding window is used, does not provide an artefact-free representation of fast dynamic processes. In general, the EPIK acquisition scheme affords a substantial increase in the signal-to-noise ratio per unit time in comparison with both single-shot and multi-shot EPI techniques because, effectively, it oversamples the central part of $k$-space and undersamples the periphery. The above considerations led to the conclusion that EPIK may be applicable to dynamic, contrastenhanced perfusion studies.

The work presented here focuses on the further development of the EPIK sequence to provide dual contrast, that is to deliver both $T_{1}$ - and $T_{2}^{*}$-weighted images without the application of additional radiofrequency pulses. The first challenge in this endeavour is to establish $T_{1}$-dominant image contrast, which requires a considerable shortening of the long echo time typical for all EPI-based techniques. The second problem to be resolved is the need to further accelerate the EPIK readout to be able to acquire data for an additional image following the same radiofrequency excitation. Both the above-mentioned complications can be resolved simultaneously by combining the EPIK readout scheme with half-Fourier reconstruction. Obviously, since the application of half-Fourier reconstruction affords a substantial time saving, the EPIK readout is shortened enabling the acquisition of data for a second gradient echo image. The requirement that the echo time be approximately equal to $T_{2}^{*}$ results in an optimum contrast-to-noise ratio in the second, $T_{2}^{*}$-weighted image. The goal of establishing predominantly $T_{1}$ contrast for the first image can be accomplished if $k$-space traversal starts with the acquisition of lines which are close to the centre of $k$-space. The echo time is thus defined by the number of additional lines required for half-Fourier reconstruction and this can be made reasonably short.

In this work, a new pulse sequence, dual-contrast EPIK (DC-EPIK), is presented, the characteristics of the method are discussed and the general applicability of the technique is demonstrated. Since two images are inherently acquired with different echo times, $T_{2}^{*}$ maps can be estimated. Further, through the judicious application of the sequence with two different flip angles, $T_{1}$ maps can also be estimated (Imran et al 1999). It is explicitly noted here that the purpose of the sequence is not fast and accurate $T_{1}$ and $T_{2}^{*}$ mapping but rather an estimation of these quantities dynamically following the injection of contrast agent. Detailed discussions of the data processing of the acquired images are not relevant here and have therefore been omitted.

\section{Theory}

\section{Reconstruction of parametric maps}

The signal intensity of EPIK for the case that TR is smaller than, or comparable to, the $T_{1}$ of interest is well described by the signal equation (Haacke et al 1999) for spoiled gradient echo imaging techniques

$$
S \propto M_{0} \frac{1-\mathrm{e}^{-\frac{\mathrm{TR}}{T_{1}}}}{1-\cos \alpha \cdot \mathrm{e}^{-\frac{\mathrm{TR}}{T_{1}}}} \cdot \sin \alpha \cdot \mathrm{e}^{-\frac{\mathrm{TE}}{T_{2}^{*}}},
$$

where $M_{0}$ is the equilibrium magnetization, $T_{1}$ and $T_{2}^{*}$ are the relaxation times and $\alpha$ is the flip angle used in the sequence. Isolating $T_{2}^{*}$ effects and assuming a single-exponential $T_{2}^{*}$ decay, it is possible to rewrite the signal intensity of the image pixel as

$$
S(x, y)=S_{0}(x, y) \mathrm{e}^{-\frac{\mathrm{TE}}{T_{2}^{*}(x, y)}}=S_{0}(x, y) \mathrm{e}^{-\mathrm{TE} \cdot R_{2}^{*}(x, y)}
$$


where $S_{0}(x, y)$ is the hypothetical signal intensity at TE $=0$ and $R_{2}^{*}=1 / T_{2}^{*}$ is the effective transverse relaxation rate. For a dual-contrast technique, the relaxation rate can be estimated from the ratio of the pixel intensities of two images:

$$
R_{2}^{*}=-\frac{\ln \left(S_{2} / S_{1}\right)}{\mathrm{TE}_{2}-\mathrm{TE}_{1}},
$$

where $\mathrm{TE}_{1}$ and $\mathrm{TE}_{2}$ are the echo times of the first and second echoes, and $S_{1}$ and $S_{2}$ are the pixel signal intensities, respectively. Having calculated $R_{2}^{*}$ maps according to equation (3), it is possible to reconstruct images corresponding to $\mathrm{TE}=0$ as follows:

$$
S_{0}(x, y)=S(x, y) \mathrm{e}^{\mathrm{TE} \cdot R_{2}^{*}(x, y)} .
$$

The images thus produced contain no $T_{2}^{*}$ weighting. Comparing equations (1) and (2), it is easy to see that

$$
S_{0} \propto \frac{1-\mathrm{e}^{-\frac{\mathrm{TR}}{T_{1}}}}{1-\cos \alpha \cdot \mathrm{e}^{-\frac{\mathrm{TR}}{T_{1}}}} \cdot \sin \alpha
$$

and hence is suitable for estimating $T_{1}$ relaxation times. Indeed, comparing the extrapolated $\mathrm{TE}=0$ image intensities from the two images acquired with different flip angles, $\alpha$ and $\beta$, it is possible to reconstruct values of $T_{1}$ for each image pixel. By substituting $\alpha$ and $\beta$, into equation (5) and through straightforward algebraic manipulation, $T_{1}$ for each pixel can be obtained according to the formula:

$$
T_{1}=-\frac{\mathrm{TR}}{\ln \left(\frac{S_{\alpha} / S_{\beta} \sin \beta-\sin \alpha}{S_{\alpha} / S_{\beta} \cos \alpha \sin \beta-\cos \beta \sin \alpha}\right)},
$$

where $S_{\alpha}$ and $S_{\beta}$ are extrapolated TE $=0$ image intensities acquired with the two flip angles, $\alpha$ and $\beta$, respectively. In practical implementations of this method for $T_{1}$ mapping (Imran et al 1999), it is important to be aware of the accuracy (approximately $\pm 2 \%$ for grey and white matter at $0.5 \mathrm{~T}$ (Imran et al 1999)) limited dynamic range of the sample $T_{1}$ values. When selecting the sequence repetition time, TR, one has to keep in mind that the condition $\mathrm{TR} \ll T_{1}$ limits SNR in the source images, whilst TR $\geqslant 3 T_{1}$ creates an insufficiently small amount of $T_{1}$ weighting.

\section{Methods}

\section{Pulse sequence}

The approach presented in this paper combines the keyhole method with a continuous, interleaved update of the high spatial-frequency information (figure 1). In contrast to the original keyhole method (van Vaals et al 1993, Jones et al 1993), the proposed technique is based on EPI and results from the further development of the original EPIK method (Shah and Zilles 2003, Zaitsev et al 2001). The additional features incorporated into the new sequence are: (a) a modified readout scheme to enable half-Fourier data acquisition and reconstruction and (b) acquisition of dual-contrast (multiple-contrast) images.

As in the original EPIK sequence (figure 1(a)), half-Fourier EPIK (figure 1(b)) combines an extensively sampled central part of $k$-space with a sparsely sampled periphery but because of the asymmetric $k$-space trajectory, readout starts directly with acquisition of the keyhole portion, skipping the top sparse region of original EPIK. Data acquisition begins several lines above the centre of $k$-space rather than directly from the centre. These additional lines are commonly referred to as 'overscan' lines and are required to calculate a low-resolution phase 


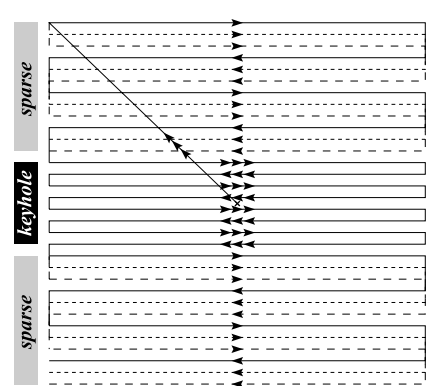

(a)

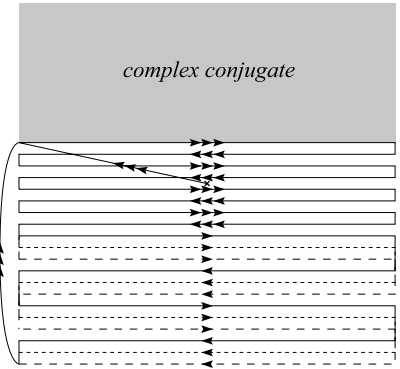

(b)

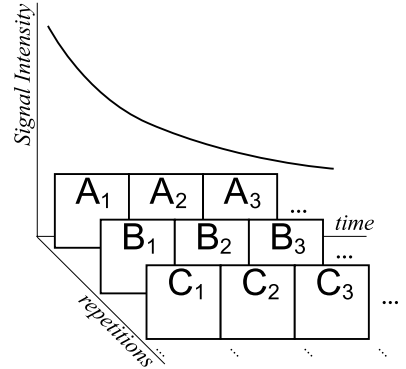

(c)

Figure 1. (a) Schematic representation of the $k$-space trajectory for the EPIK sequence with a SPARSE factor of 3. Standard, 3-shot EPIK is defined as $\left(A_{1}+B_{1}+C_{1}\right)$ and yields three images with the same contrast (TE) but each with its independent keyhole. The $k$-space trajectory of DC-EPIK also with a SPARSE factor of 3 is depicted in (b). DC-EPIK is defined as $\left(\mathrm{A}_{1}+\mathrm{B}_{1}+\right.$ $\left.C_{1}\right)$ and $\left(A_{2}+B_{2}+C_{2}\right)$ with contrasts defined by $T_{1}$ and $T_{2}$; the result is six images, again each with its independent keyhole. Here, a $k$-space plane is sampled in three distinct parts, a central keyhole where $\Delta k_{\mathrm{y}}=1 / \mathrm{FOV}$, a SPARSE region where $\Delta k_{\mathrm{y}}{ }^{\prime}=3 \times 1 / \mathrm{FOV}$ and a region where the data are calculated from the SPARSE region. Each subsequent shot begins with the first $k$-space line of the keyhole region following which different lines in the SPARSE region are read. Following multiple acquisitions equal to the SPARSE factor, all $k$-space has been sampled with the keyhole region sampled three times. By sharing the SPARSE data, three separate images can be constructed. Following putative radiofrequency pulses, the relationship of the signal decay and acquisition of multiple contrasts $(1,2,3, \ldots)$ in multiple shots $(\mathrm{A}, \mathrm{B}, \mathrm{C}, \ldots)$ is shown in (c). It is important to note that no data sharing takes place between images with different contrasts, that is, along the 'time' axis.

map which is essential for half-Fourier reconstruction. Acquisition of the keyhole part is followed by a sparse region, which is merely a fragment of an interleaved EPI readout with an interleave, or sparse, factor of $s$. As in the original EPIK sequence (Shah and Zilles 2003, Zaitsev et al 2001), the nomenclature for the keyhole factor used here is $k$, that is, $k$ is the inverse of the fraction of $k$-space comprising the keyhole, e.g. if the keyhole covers $\frac{1}{4}$ of $k$-space, then $k=4$. In order to define the acquisition matrix for half-Fourier EPIK, assume a general $k$-space acquisition which is $N \times N$ points. Further, let $\operatorname{EPIK}\left\langle n_{\mathrm{ov}}, k, s, i\right\rangle$ be the sampling scheme where $n_{\mathrm{ov}}$ is the number of overscan lines, $k$ is the keyhole factor, $s$ is the sparse factor and $i$ is the index of the interleaf $(0 \leqslant i<s)$. A generalized EPIK \langle\rangle sampling scheme may thus be defined as follows:

$$
\begin{gathered}
\operatorname{EPIK}\left\langle n_{\mathrm{ov}}, k, s, i\right\rangle=\left[n_{\mathrm{ov}}, n_{\mathrm{ov}}-1, n_{\mathrm{ov}}-2, \ldots, 0,-1, \ldots,-\frac{N}{2 k}+2,-\frac{N}{2 k}+1, \ldots,\right. \\
\left.-\frac{N}{2}+3 s-i,-\frac{N}{2}+2 s-i,-\frac{N}{2}+s-i\right]
\end{gathered}
$$

and the accompanying expression for the acquisition time for one readout module, TA, is given by

$$
\mathrm{TA}=\Delta t\left(n_{\mathrm{ov}}+N \frac{s+k-1}{2 \cdot s \cdot k}\right),
$$

where $\Delta t$ is the readout time per line of $k$-space. From equations (7) and (8), different schemes to cover $k$-space may be devised and the concomitant time saving may be calculated. This paper describes the particular implementation of a dual-contrast sequence with the SPARSE factor $s=3$, keyhole factor $k=4$ and number of overscan lines $n_{\mathrm{ov}}=16$ and matrix size $N=128$. 


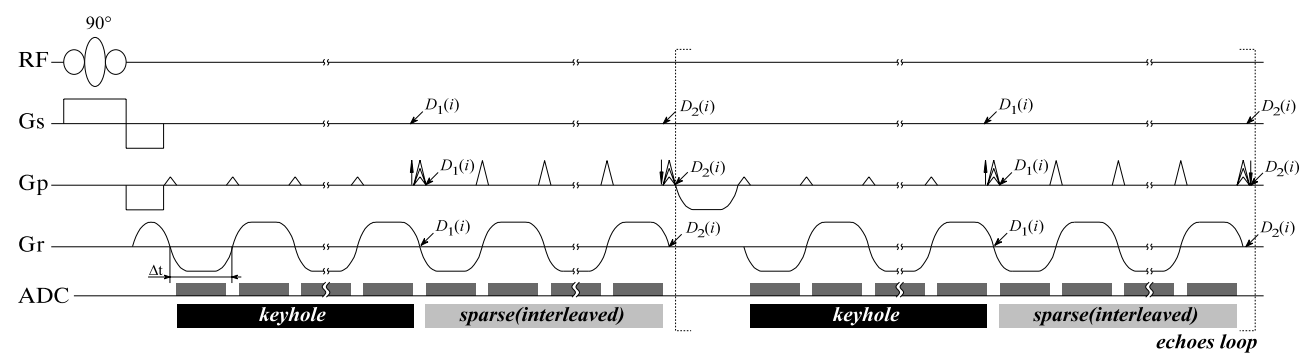

Figure 2. Schematic representation of a generic sequence timing diagram for the multiple-contrast, half-Fourier EPIK method. Specifically, for DC-EPIK, each readout gradient had a sinusoidal ramp of duration $300 \mu \mathrm{s}$ and a flat-top of $280 \mu \mathrm{s}$. Ramp sampling on sinusoidal ramps was used with a nonlinear ADC sample raster. Line readout time, $\Delta t$, was equal to $880 \mu \mathrm{s}$. The various time delays required for echo time shifting are also shown. Spoiling gradients, which have been omitted for the sake of clarity, were applied after acquisition of the second SPARSE region.

Notwithstanding the above, the flexibility of the method to acquire multiple contrasts is shown in figure 1(c). Here, the numerical subscript represents different contrasts with increasing $T_{2}^{*}$ and the letters represent separate shots. Note that although in our implementation $n_{\mathrm{ov}}$ coincided with half the keyhole size, $1 / 2(N / k)$, it is by no means a requirement. The number of overscan lines is selected based on the level of the accepted half-Fourier reconstruction artefacts and typically lies between 8 and 16 for a $128^{2}$ image matrix.

Due to the reduced sampling of high $k$-space regions, additional reconstruction steps are required to fill the missing information. A number of alternative approaches which may be used are: (a) data sharing or sliding window techniques to share high-frequency information in the time series of acquired images; (b) a priori information about the location and character of expected intensity changes to perform interpolation and (c) parallel acquisition techniques in combination with phased array receiver coils. In the current implementation, the data sharing approach has been chosen because of its simplicity, robustness, computational ease and operator independence. In the chosen approach, missing $k$-space data were shared from the neighbouring shots in the time series. Using both 'past' and 'future' neighbours was found to provide a better representation of the fast dynamic processes occurring during the bolus passage.

Figure 2 is a schematic representation of the multi-contrast, half-Fourier EPIK pulse sequence. The sequence consists of standard slice selection, an initial traversal to the outermost point of the keyhole, followed by two equivalent readout modules, where the second module is preceded by a large phase-encode gradient applied to jump from the bottom of $k$-space to the beginning of the keyhole. The dashed brackets represent the position of a possible multi-contrast loop which extends the sequence to acquire more than two gradient echoes. This, however, was not carried out as part of the work reported here because of a restrictive pulse-sequence programming environment. Echo time shifting delays (McKinnon 1993, Butts et al 1994) play an important role in the production of artefact-free images and are denoted by $D_{1}$ and $D_{2}$ in figure 2 ; these were implemented as described by Zaitsev et al (2001).

Reconstruction of ghost-free images required phase correction between the odd and even gradient echoes to ameliorate the effects of readout gradient reversals. A non-phase-encoded calibration scan was performed prior to the actual acquisition to calculate a phase correction vector on a pixel-by-pixel basis. Application of first-order phase correction as well as image-based phase correction techniques (Buonocore and Zhu 2001, Hennel 1999) was found 
to be inadequate because of the poor phase response of the analogue filters installed in the scanner.

\section{Partial Fourier reconstruction}

Partial Fourier reconstruction was first proposed in the early years of MRI (Margosian 1985). Since then a multitude of half-Fourier techniques have been developed and successfully incorporated into different imaging methods. However, application of halfFourier reconstruction to imaging methods where phase errors are dominant, such as gradient echo imaging and EPI in particular, is a non-trivial task. Indeed, all half-Fourier reconstruction algorithms rely on the assumption that the reconstructed image is purely real, or can be made real by phase correction with a low resolution phase map. Typically, a few overscan lines are acquired to produce such a map. The above considerations assume that image phase errors have a low spatial resolution; this is not true in the case of echo planar imaging, where these errors can be rather local. In contrast to Jesmanowicz et al (1998), in order to produce reasonable quality images, it was necessary here to acquire $12-16$ overscan lines for a $128 \times$ 128 image matrix and to use the most advanced and computationally expensive reconstruction techniques, projection onto convex sets (POCS) (Haacke et al 1991, McGibney et al 1993). Compared with the POCS method used here, images provided by simpler methods such as conjugate synthesis were considered to be of unacceptable quality.

\section{Implementation}

A multi-slice, dual-contrast, half-Fourier EPIK pulse sequence was implemented on a Siemens Magnetom Vision 1.5T whole-body scanner (Siemens Medical Systems, Erlangen, Germany) equipped with a standard gradient system providing $25 \mathrm{mT} \mathrm{m}^{-1}$ peak strength with $300 \mu \mathrm{s}$ risetime and sinusoidal ramps. Ramp sampling on the sinusoidal ramps was used along with a nonlinear ADC sample raster and a dwell time of $8 \mu \mathrm{s}$ on the flat-top (Schmitt et al 1998); this gives a readout bandwidth of $1250 \mathrm{~Hz} /$ pixel. Line readout time was equal to $880 \mu \mathrm{s}$ and therefore with 48 lines, this results in a bandwidth of $23.7 \mathrm{~Hz} /$ pixel in the phase-encode direction. The sequence provided a matrix size of $128 \times 128$ in the reconstructed images. No zero filling was performed. A $2.56 \mathrm{~ms}$ sinc pulse was used for slice-selective excitation. The current implementation acquired 16 overscan lines which resulted in minimum TEs of $18 \mathrm{~ms}$ and $62 \mathrm{~ms}$ for the first and second images, respectively. Excitation of each slice was preceded by a routine 1-3-3-1 volume fat suppression module (Schmitt et al 1998). Because of platform hardware/software limitations, the sequence was only capable of providing limited volume coverage restricted to four slices. It is important to note that this restriction is by no means intrinsic to the method, but rather reflects the shortcomings of the scanner software.

\section{Phantom studies}

In order to test sequence performance, phantom experiments were carried out prior to imaging healthy volunteers. Phantom experiments included comparison of the sequence against EPI, half-Fourier EPI and the original, full $k$-space EPIK technique. Sequence parameters for halfFourier, DC-EPIK were $\mathrm{TE}_{1} / \mathrm{TE}_{2} / \alpha=18 \mathrm{~ms} / 62 \mathrm{~ms} / 90^{\circ}$, FOV $=200 \mathrm{~mm}$, matrix size $=$ $128 \times 128$, slice thickness $=4 \mathrm{~mm}$, no signal averaging. Sequence parameters for the other three sequences, such as matrix size, slice thickness and position, echo time (62 ms) and flip angle were held unchanged for the purposes of method comparison. All four sequences employed the same readout gradient waveform and bandwidth. A resolution phantom containing a series of different-sized plastic rods in doped water was imaged. 
In vivo imaging experiments

In order to verify image quality and to test the dual-contrast capabilities of EPIK, healthy volunteers were imaged. Sequence parameters were $\mathrm{TE}_{1} / \mathrm{TE}_{2}=18 \mathrm{~ms} / 62 \mathrm{~ms}, \mathrm{FOV}=$ $210 \mathrm{~mm}$, matrix size $=128 \times 128$, slice thickness $=6 \mathrm{~mm}$, no signal averaging. The repetition time TR was set to three different values: $1 \mathrm{~s}, 2 \mathrm{~s}, 3 \mathrm{~s}$; in each experiment the flip angle, $\alpha$, was chosen according to the Ernst formula to maximize the signal-to-noise ratio for the grey matter $\left(67^{\circ}, 82^{\circ}, 87^{\circ}\right.$, respectively). The gradient waveforms, readout bandwidth and sampling parameters were as those described above. Volunteer measurements were performed in accordance with IRB regulations. Informed consent was obtained prior to the experiment.

\section{Clinical studies}

For initial in vivo clinical studies, sequence parameters were: $\mathrm{TE}_{1} / \mathrm{TE}_{2} / \alpha=18 \mathrm{~ms} / 62 \mathrm{~ms} /$ $65^{\circ}$; matrix size $=128 \times 128$; four slices; slice thickness $=8 \mathrm{~mm}$; no signal averaging. The same sequence was used to acquire proton-density weighted images prior to the dynamic study; all sequence parameters were left unchanged except for $\alpha$, which was $10^{\circ}$, and nine acquisitions were averaged. These images enabled $T_{1}$ maps to be calculated according to equation (6). The DC-EPIK images were obtained at a rate of $1 \mathrm{~Hz}(\mathrm{TR}=1 \mathrm{~s})$ and a total of 96 images were acquired for each contrast. The first three images were discarded because of $T_{1}$ saturation effects. Following acquisition of a $10 \mathrm{~s}$ baseline, gadopentatate dimeglumine (Magnevist; Schering Healthcare, Burgess Hill, UK) was injected intravenously as a bolus through a peripherally placed cannula $\left(0.1 \mathrm{mmol} \mathrm{kg}^{-1}\right.$ body weight, using an automated power injector at a rate of $4 \mathrm{ml} \mathrm{s}^{-1}$ and followed by a $20 \mathrm{ml}$ flush of normal saline).

Initial studies not reported here had demonstrated that the changes in tissue $T_{1}$ upon accumulation of the contrast agent were more dramatic than had been anticipated, that is, the selected TR of $1 \mathrm{~s}$ did not provide sufficient dynamic range for the reconstruction of estimates of $T_{1}$ maps. For subsequent clinical studies, the sequence parameters were modified in the following way: image acquisition rate $2 \mathrm{~Hz}(\mathrm{TR}=500 \mathrm{~ms}) ; \alpha=55^{\circ}$; matrix size $=$ $128 \times 128$; 3 slices; total number of images was 180 for each contrast.

\section{Data analysis}

Images acquired with DC-EPIK were used to estimate maps of the relaxation parameters as described briefly in the theory section of this paper. Prior to these calculations, a spatial 2D Gaussian filter (2 pixels full-width-at-half-maximum) was applied to all images in order to boost the SNR and diminish effects associated with subject motion. When calculating the longitudinal relaxation time, $T_{1}$, according to equation (6), a calibration procedure was required. $T_{1}$ values calculated in this way depend critically on the exact knowledge of the excitation flip angles used. In practice, these flip angles deviate from the values specified in the sequence measurement protocol due to slice-profile imperfections and $\mathbf{B}_{1}$ inhomogeneities. The latter, however, are mostly sample independent at the moderate field strength as $1.5 \mathrm{~T}$, which enabled the use a phantom calibration. The calibration procedure included imaging of a multi-compartment phantom with known $T_{1}$ values, followed by fitting of the resultant data to the model given by equation (6) with the values of flip angles left as fitting parameters. Values of flip angles acquired in this way were then used for the reconstruction of in vivo $T_{1}$ maps.

Perfusion analysis was performed according to the methods described by Barbier et al (1999) where signal intensities from the long and short echoes were combined to produce maps of $\Delta R_{2}^{*}$, which eliminates the undesirable ' $T_{1}$ shine through' effect commonly seen 


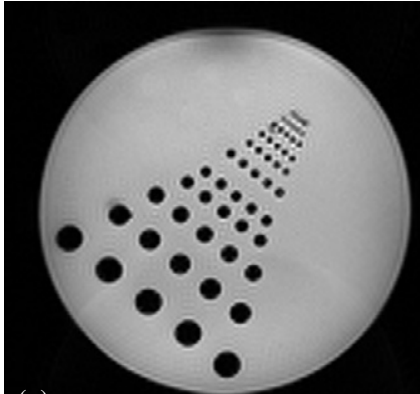

(a)

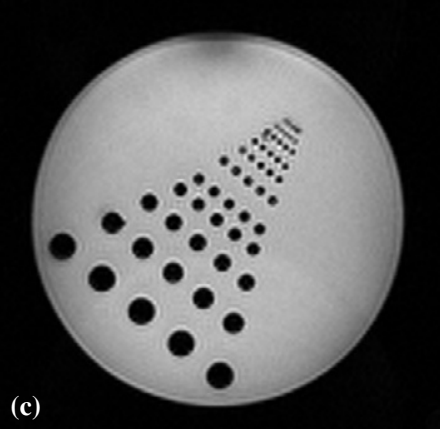

(b)
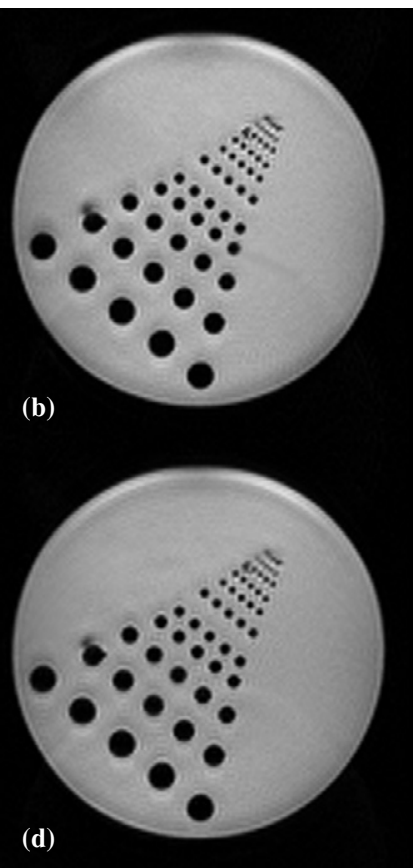

Figure 3. Images through a resolution phantom acquired with four different sequences: (a) full $k$-space EPI; (b) half-Fourier EPI; (c) full $k$-space EPIK and (d) the second echo from DC-EPIK. As seen from visual inspection, there is very little loss of quality between half-Fourier EPI and half-Fourier EPIK. Furthermore, apart from the typical half-Fourier artefacts (hypo-intense regions near the second-largest row of rods), there is little to distinguish full $k$-space EPI from DC-EPIK.

in susceptibility-contrast techniques. Gamma variate functions were fitted to the $\Delta R_{2}^{*}$ time courses using a Levenberg-Marquardt algorithm. The gamma variate function is the theoretical response to an ideal rectangular bolus. The real bolus is not ideal, and since it was not possible, with this particular implementation of DC-EPIK, to measure the true arterial input function, only maps of relative regional cerebral blood volume ( $\mathrm{rCBV}$ ), relative mean transit time (rMTT) and relative regional cerebral blood blow (rrCBF) were calculated from the fitted data. Images were spatially smoothed prior to analysis using $3 \times 3$ boxcar filter. Thereafter, a temporal UNFOLD (Madore et al 1999) filter (parameters: $E_{f}=0.25, k T=0.04$ ) was applied in order to minimize effects of aliasing arising from undersampling of temporal data.

\section{Results}

Figure 3 shows images of the standard resolution phantom acquired with four different sequences: (a) standard full $k$-space EPI; (b) half-Fourier EPI; (c) original full $k$-space EPIK and (d) the second echo from DC-EPIK. All four images were acquired under similar conditions where the echo time was fixed to $62 \mathrm{~ms}$. No degradation of image quality is observed between images (a) and (c), or between images (b) and (d). Comparison of the full $k$-space and halfFourier images reveals artefacts which are typical of half-Fourier reconstruction of gradient echo data. Nevertheless, images (b) and (d) show that these artefacts are not unacceptable and are reasonably well controlled. 

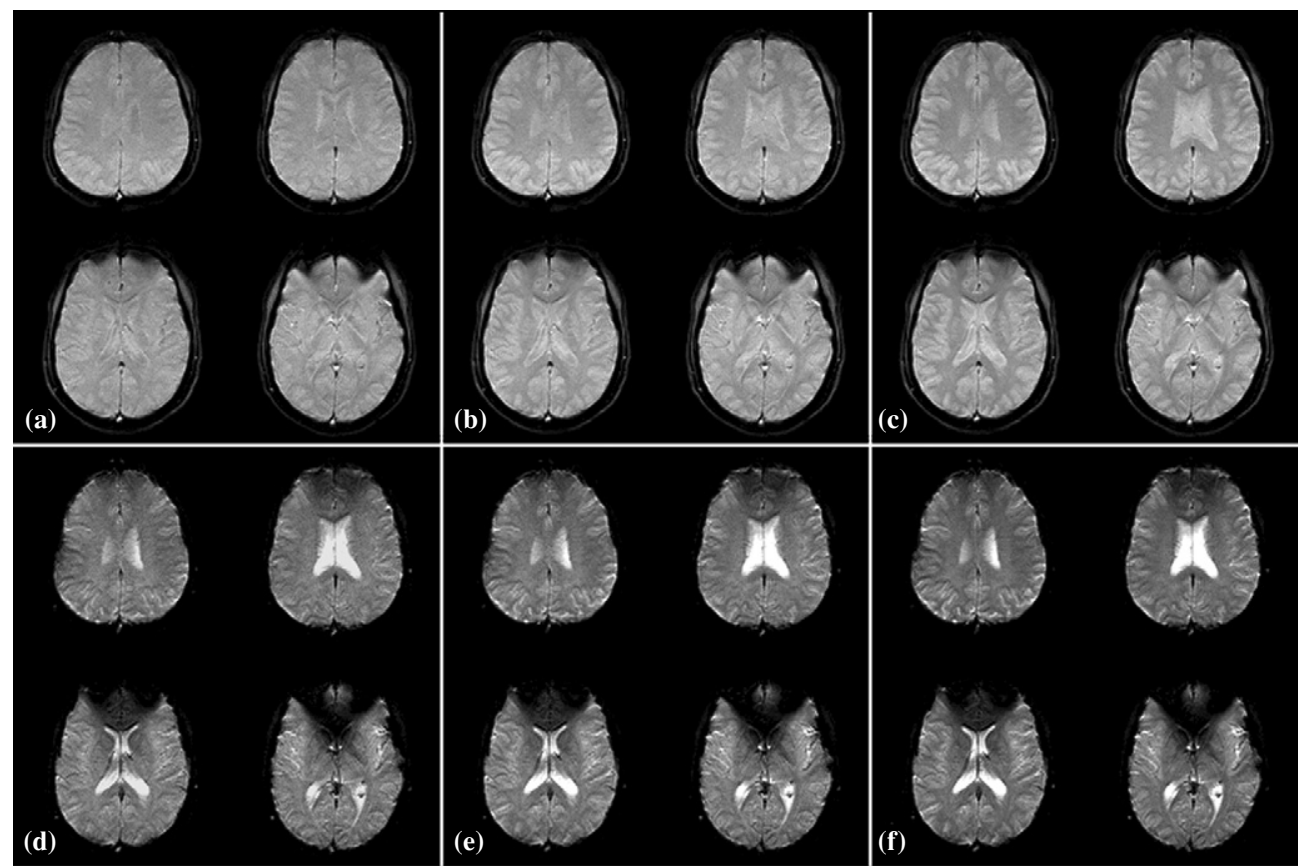

Figure 4. Four representative slices through the brain of a normal volunteer acquired with DC-EPIK: (top row) predominantly $T_{1}$-weighted images and (bottom row) predominantly $T_{2}^{*}$-weighted images acquired with different TR times of $1 \mathrm{~s}(\mathrm{a}, \mathrm{d}), 2 \mathrm{~s}(\mathrm{~b}, \mathrm{e})$ and $3 \mathrm{~s}(\mathrm{c}, \mathrm{f})$. The images demonstrate the high quality as well as the dual-contrast capabilities of DC-EPIK in vivo.

Figure 4 demonstrates the image quality as well as the dual-contrast capabilities of DC-EPIK in vivo. Four slices through the brain of a normal, healthy volunteer are presented for three different values of TR, demonstrating a varying amount of $T_{1}$ weighting. The high image quality and substantially different contrasts between these images should be noted.

Preliminary clinical results are presented in figures 5 and 6 . Figures 5(a) and (b) demonstrate the ability of DC-EPIK to track changes in tissue $T_{1}$ times with high temporal and spatial resolution. A $T_{1}$ map, calculated according to equation (6) and using the proton density-weighted image acquired prior to the injection of contrast agent, from the fourslice dynamic dataset is depicted in figure 5(a). Measurement parameters were as follows: $\mathrm{TR}=1 \mathrm{~s}, \mathrm{TE}_{1}=18 \mathrm{~ms}, \mathrm{TE}_{2}=62 \mathrm{~ms}, \alpha_{1}=65^{\circ}, \alpha_{2}=65^{\circ}$, matrix size $=128^{2}$, four slices, slice thickness $=8 \mathrm{~mm}$, no signal averaging. The evolution of the $T_{1}$ values from three regionsof-interest $(3 \times 3$ pixels $)$ is plotted in figure $5(\mathrm{~b})$. $\mathrm{ROI}_{1}$ was positioned in an area of moderate contrast agent accumulation. Fitting of the corresponding curve to a pharmacokinetic model would, in principle, enable quantification of tissue contrast uptake parameters. However, as seen from the plot for $\mathrm{ROI}_{2}$, the scale of $T_{1}$ changes was underestimated when selecting sequence parameters, especially the sequence repetition time. Indeed, the image intensity is sensitized to changes in $T_{1}$ through not allowing full recovery between successive repetition cycles. However, with the chosen TR $=1 \mathrm{~s}$ for tissue components with $T_{1} \leqslant 250 \mathrm{~ms}$, the recovery of longitudinal magnetization is nearly complete. The presence of noise makes the calculation of $T_{1}$ based on image intensities according to equation (6) unfeasible. In subsequent clinical experiments, TR was reduced to $500 \mathrm{~ms}$ to enable the quantification of the longitudinal relaxation time for faster-relaxing tissues. 


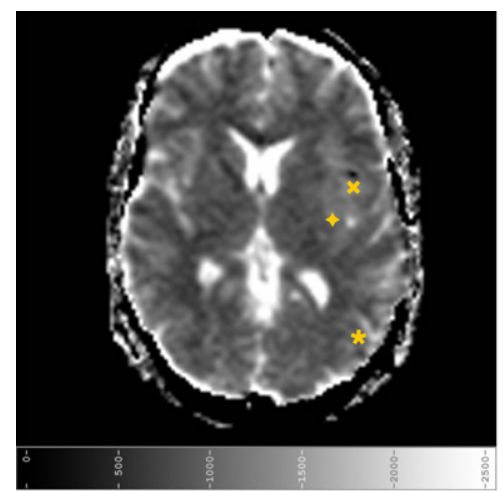

(a)

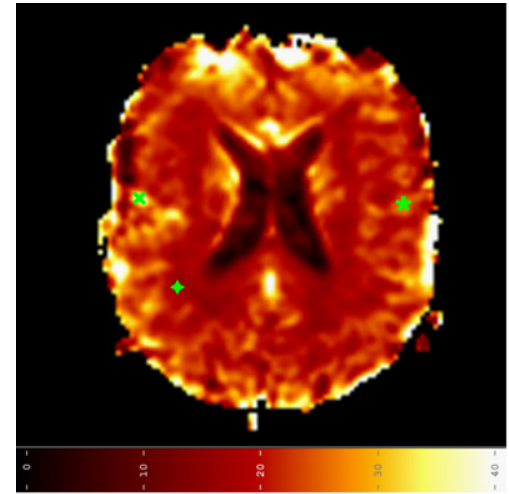

(c)

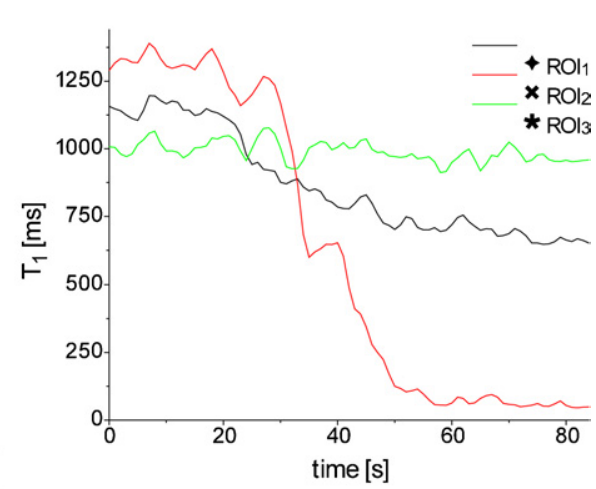

(b)

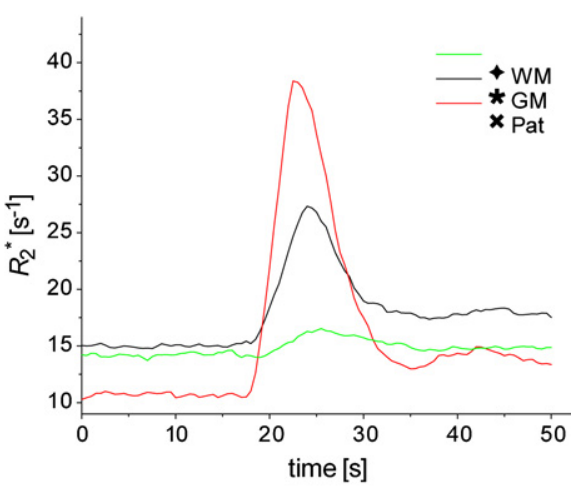

(d)

Figure 5. (a) A single-slice $T_{1}$ map, calculated from the four-slice dynamic series. The $T_{1}$ map presented corresponds to the pre-contrast condition. (b) Evolution of tissue $T_{1}$ values following contrast agent injection for three small $(3 \times 3$ pixels $)$ regions of interest. The regions of interest are marked with the respective symbols in (a). Tracking the dynamics of the accumulation of the contrast agent in $\mathrm{ROI}_{1}$ and $\mathrm{ROI}_{2}$ would, in principle, allow calculation of tissue permeability parameters. $\mathrm{ROI}_{3}$ corresponds to the healthy grey matter and shows no accumulation. (c) Transverse relaxation rate map and (d) evolution during the passage of the bolus of contrast agent. The $R_{2}^{*}$ map in (c) was calculated from the three-slice dataset and corresponds to the moment of bolus passage. The regions-of-interest for the $R_{2}^{*}$ versus time plots (d) are marked with the corresponding symbols. The data shown in (d) are sufficient to calculate regional haemodynamic parameters such as blood volume and flow.

Figures 5(c) and (d) show the passage of the bolus of the contrast agent as seen via observation of changes in $R_{2}^{*}$. Figure $5(\mathrm{c})$ is an $R_{2}^{*}$ map of a transverse slice through the brain of a patient with a suspected tumour, taken from the dynamic series at a time which corresponds to the passage of the bolus. Measurement parameters were as follows: $\mathrm{TR}=500 \mathrm{~ms}, \mathrm{TE}_{1}=18 \mathrm{~ms}, \mathrm{TE}_{2}=62 \mathrm{~ms}, \alpha=55^{\circ}$, matrix size $=128^{2}$, three slices, slice thickness $=8 \mathrm{~mm}$, no signal averaging. Relaxation rate maps were reconstructed from the ratio of the magnitude images according to equation (3). An additional pre-processing step was required prior to calculation of the parametric maps which included the removal of the temporal frequency component oscillating at exactly $1.5 \mathrm{~Hz}$, the frequency of repetition of exactly the same interleaf in the SPARSE region. This was accomplished via a pixel-by-pixel Fourier-transformation of the dynamic dataset in the temporal dimension, followed by the 


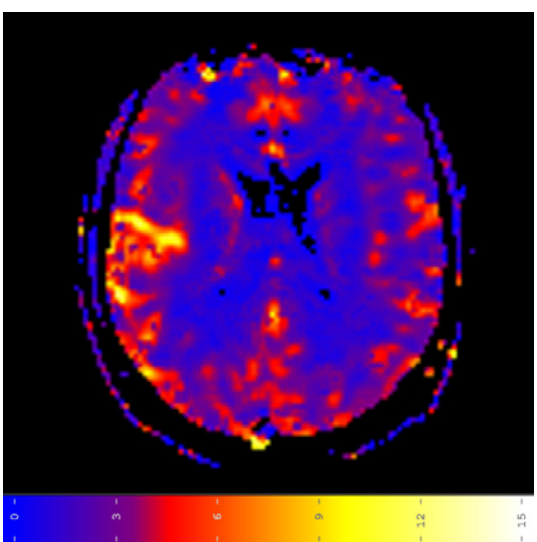

(a)

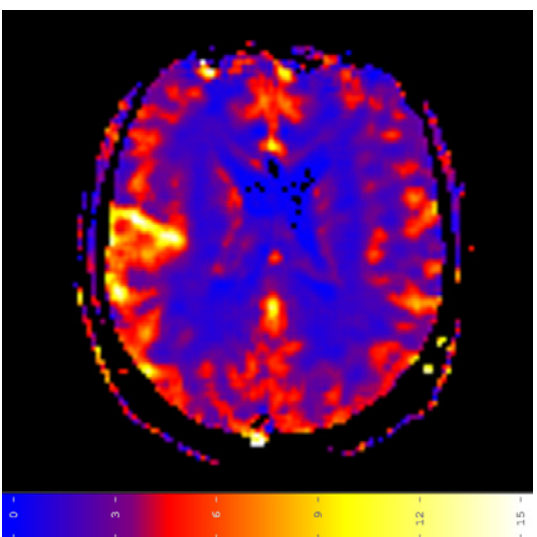

(b)

Figure 6. Functional maps calculated from the dynamic acquisition performed on a patient with a suspected tumour. A calculated relative regional cerebral volume (rrCBV) map is shown in (a) and relative regional cerebral flow (rCBF) in (b). For both $\mathrm{rCBV}$ and $\mathrm{rCBF}$ values have been normalized to white matter.

replacement of the corresponding frequency components with the geometric average of the neighbouring frequency components.

Figure 5(d) contains plots of $R_{2}^{*}$ as a function of time for three representative regions-ofinterest. As can be seen, changes in the tissue $R_{2}^{*}$ are more significant in grey matter than in white matter, reflecting the fact that the grey matter is characterized by a larger regional blood volume. The third region-of-interest was positioned in an area of pathologically increased blood volume and flow. From the information presented in figures 5(c) and (d), the contrast agent concentration versus time dependence can be calculated for each image pixel followed by the fitting of the acquired data to the gamma variate function with subsequent integration in order to produce maps of haemodynamic parameters as described above.

Figure 6 shows the reconstructed relative regional cerebral blood volume and flow maps for the same subject as in figures 5(c) and (d). The ratio of the first and second contrasts of the DC-EPIK acquisition was used to create $R_{2}^{*}$ curves without the confounding ' $T_{1}$ shine-through' effect. These curves were used to calculate the concentration versus time dependences which were then fitted to a gamma variate function in order to reduce noise in the resulting maps. Functional maps were reconstructed by integrating the fitted gamma variate functions for each image pixel.

\section{Discussion}

The use of DC-EPIK for the investigation of time-dependent phenomena, such as dynamic studies of contrast agent uptake, offers a number of advantages over conventional methods. By virtue of data sharing, a higher apparent temporal resolution is achieved which transpires to be a real gain if the contrast changes being tracked take place on a time scale which is slower than the image update rate. The spatial resolution of DC-EPIK is as expected for single-contrast EPIK, that is, it is superior to that of EPI (Shah and Zilles 2003, Zaitsev et al 2001). Although it has not been explicitly shown here, DC-EPIK offers a degree of flexibility in that the SPARSE factor, the keyhole factor and the oversampling factor are adjustable parameters which influence the temporal resolution and the achievable degree of immunity 
from susceptibility artefacts. Because DC-EPIK intrinsically acquires two images, registration of these images is inherent; this is, of course, applicable to other dual echo sequences (Barbier et al 1999, Miyati et al 1997, Vonken et al 2000) also, but DC-EPIK is faster since it is based on EPI rather than FLASH. The acquisition of an independent keyhole during each shot ensures that the contrast-to-noise of each reconstructed image is, to all practical intents, an independent measure. Thus, the time series of data acquired in experiments such as those reported here constitutes a finer sampling of the dynamic processes under investigation; this finer sampling leads to more accurate fitting to a given model and, if the arterial input function is known, better quantitation. The speed of EPI leads to a sequence which is multi-slice and, because larger radiofrequency pulse flip angles than in FLASH are employed, provides higher SNR than in FLASH-based sequences.

The disadvantages of DC-EPIK are, first and foremost, the same as those afflicting interleaved EPI and standard EPIK. These include signal dropout in areas of high magnetic field heterogeneity and sensitivity to inter-shot patient motion. The loss of temporal resolution of DC-EPIK compared with EPI is not a serious issue since the sequence proposed here is still faster than most other competing techniques. The use of half-Fourier reconstruction and the sliding window does result in a time penalty during image reconstruction. This is an issue which is particularly acute on older scanners, although, in mitigation, even here reconstruction may be performed offline.

Although the number of lines read in each shot of DC-EPIK is $3 / 8$ that of a standard EPI readout, through echo-sharing and half-Fourier reconstruction, DC-EPIK does not suffer from a significant reduction of image quality since the full matrix size is maintained. Indeed, given that the bandwidth of EPIK and DC-EPIK in the phase encoding direction is concomitantly larger than that of EPI, signal loss and geometric distortions due to susceptibility effects should be more circumscribed.

The sequence described in this paper used a SPARSE factor of 3, a keyhole factor of 4 and $n_{\mathrm{ov}}=16$. However, as already noted, the DC-EPIK sequence offers ample opportunity to vary these parameters to suit the experimental conditions. A significant consequence of this flexibility is that more than two images may be acquired, that is, extension of the sequence to triple contrast or more. By reducing the $n_{\mathrm{ov}}$ factor, not only is there a concomitant time saving, but also the TE of the first echo may be reduced. Simultaneous acquisition of the arterial input function should also enable the sequence to provide quantitative measures of, for example, regional blood flow, blood volume, tissue perfusion and permeability as well as other important physiological parameters.

An important consideration at this juncture is the accuracy of the $T_{1}$ map derived from equation (6) which is known to be circumscribed (Imran et al 1999), especially if care is not taken to measure the linearity of the RF transmitter. Although there are a number of methods for fast $T_{1}$ mapping (Shah et al 2001, Tofts 2003) that are able to deliver $T_{1}$ values with an accuracy of around 1\%, these methods can be time consuming and are therefore entirely inappropriate in the context of the type of clinical studies that are the target here. Further, if the maps are produced with a FLASH-based method, then the problem of different spatial distortions resurfaces. Thus, although the dual-angle method may not be the method of choice for accurate $T_{1}$ mapping, here, because of total acquisition time constraints, it is a justifiable compromise.

The nature of the EPIK $k$-space trajectory, and therefore by association the DC-EPIK trajectory, is such that parallel imaging is a straightforward extension. The keyhole section lends itself to two purposes; improved SNR in each reconstructed image, and correction procedures for the reconstruction of unaliased imaged from parallel imaging techniques such as GRAPPA (Griswold et al 2002). In a parallel imaging implementation of EPIK and 
DC-EPIK, $k$-space lines could be skipped in the periphery of $k$-space by a factor for parallel imaging and a factor for the sliding window reconstruction of EPIK with a concomitant speed up factor from each. It will be interesting to explore the merits of parallel acquisition of normal gradient echo methods and DC-EPIK accelerated using parallel imaging. However, more detailed examination of this issue remains to be undertaken and is outside the scope of this paper.

The preliminary results presented here show the ability of DC-EPIK to produce $T_{1}$ and $T_{2}^{*}$ relaxation time maps with relatively high spatial resolution and track both bolus passage and contrast agent accumulation with high temporal resolution. Although quantitative mapping of tissue permeability has not been demonstrated here, the results presented show that it is feasible, in principle.

\section{Conclusions}

A dual-contrast magnetic resonance imaging method, based on EPI, and incorporating elements of the keyhole technique, half-Fourier reconstruction and temporal data sharing, has been designed and implemented on a standard clinical scanner.

The quality of the images produced by the new technique was verified in phantom imaging experiments. Image quality comparisons revealed no degradation associated with the introduction of sparse sampling regions in $k$-space, indeed, the spatial resolution of the EPIK images is somewhat better. Introduction of partial Fourier reconstruction led to a degree of image quality degradation, which, however, was more pronounced for half-Fourier EPIK than for standard half-Fourier EPI. Although half-Fourier EPIK acquires only $3 / 8$ of the $k$-space lines after each excitation, phantom results show that it is able to deliver images of a quality comparable to that of standard EPI and that image artefacts are well under control. Artefact-free image reconstruction has also been demonstrated in vivo.

Initial clinical results from contrast-enhanced experiments demonstrate the ability of DCEPIK to produce simultaneous $T_{1}$ and $T_{2}^{*}$ relaxation time maps and track changes in these parameters with high temporal resolution. The ability of DC-EPIK to separate $T_{1}$ and $T_{2}^{*}$ effects makes it, in principle, applicable to quantification of perfusion in patients with a disrupted blood brain barrier. The feasibility of reconstructing high-resolution perfusion maps by tracking the first pass of the injected contrast agent bolus has been demonstrated in patients.

\section{References}

Aronen H J et al 1995 Echo-planar MR cerebral blood volume mapping of gliomas. Clinical utility Acta Radiol. 36 520-8

Barbier E L, den Boer J A, Peters A R, Rozeboom A R, Sau J and Bonmartin A 1999 A model of the dual effect of gadopentetate dimeglumine on dynamic brain MR images J. Magn. Reson. Imaging $10242-53$

Barbier E L, Lamalle L and Decorps M 2001 Methodology of brain perfusion imaging J. Magn. Reson. Imaging 13 496-520

Buonocore M H and Zhu D C 2001 Image-based ghost correction for interleaved EPI Magn. Reson. Med. 45 96-108

Butts K, Riederer S J, Ehman R L, Thompson R M and Jack C R 1994 Interleaved echo planar imaging on a standard MRI system Magn. Reson. Med. 31 67-72

d'Arcy J A, Collins D J, Rowland I J, Padhani A R and Leach M O 2002 Applications of sliding window reconstruction with Cartesian sampling for dynamic contrast enhanced MRI NMR Biomed. 15 174-83

Griswold M A, Jakob P M, Heidemann R M, Nittka M, Jellus V, Wang J, Kiefer B and Haase A 2002 Generalized autocalibrating partially parallel acquisitions (GRAPPA) Magn. Reson. Med. 47 1202-10

Haacke E M, Lindskog E D and Lin W 1991 A fast, Iterative, partial-fourier technique capable of local phase recovery J. Magn. Reson. 92 126-45 
Haacke E M, Brown R W, Thompspm M R and Venkatesan R 1999 Magnetic Resonance Imaging: Physical Principles and Sequence Design (New York: Wiley)

Hennel F 1999 Two-dimensional deghosting for EPI MAGMA 9 134-7

Imran J, Langevin F and Saint-Jalmes H 1999 Two-point method for T1 estimation with optimized gradient-echo sequence Magn. Reson. Imaging 17 1347-56

Jesmanowicz A, Bandettini P A and Hyde J S 1998 Single-shot half $k$-space high-resolution gradient-recalled EPI for fMRI at 3 Tesla Magn. Reson. Med. 40 754-62

Jones R A, Haraldseth O, Muller T B, Rinck P A and Oksendal A N 1993 K-space substitution: a novel dynamic imaging technique Magn. Reson. Med. 29 830-4

Kim E J, Kim D H, Lee S H, Huh Y M, Song H T and Suh J S 2004 Simultaneous acquisition of perfusion and permeability from corrected relaxation rates with dynamic susceptibility contrast dual gradient echo Magn. Reson. Imaging 22 307-14

Madore B, Glover G H and Pelc N J 1999 Unaliasing by Fourier-encoding the overlaps using the temporal dimension (UNFOLD), applied to cardiac imaging and fMRI Magn. Reson. Med. 42 813-28

Margosian P 1985 Faster MR imaging: imaging half the data Proc. SMRM, 4th Annual Meeting (London) p 1024

McGibney G, Smith M R, Nichols S T and Crawley A 1993 Quantitative evaluation of several partial Fourier reconstruction algorithms used in MRI Magn. Reson. Med. 30 51-9

McKinnon G C 1993 Ultrafast interleaved gradient-echo-planar imaging on a standard scanner Magn. Reson. Med. 30 609-16

Miyati T, Banno T, Mase M, Kasai H, Shundo H, Imazawa M and Ohba S 1997 Dual dynamic contrast-enhanced MR imaging J. Magn. Reson. Imaging 7 230-5

Schmitt F, Stehling M K and Turner R 1998 Echo-Planar Imaging: Theory, Technique and Application (Berlin: Springer)

Shah N J, Zaitsev M, Steinhoff S and Zilles K 2001 A new method for fast multislice T(1) mapping Neuroimage 14 1175-85

Shah N J and Zilles K 2003 Verfahren zur Untersuchung eines Objekts German patent no DE 19962 845. 9-33

Tofts P 2003 Quantitative MRI of the Brain (Chichester: Wiley)

van Vaals J J, Brummer M E, Dixon W T, Tuithof H H, Engels H, Nelson R C, Gerety B M, Chezmar J L and denBoer J A 1993 'Keyhole' method for accelerating imaging of contrast agent uptake J. Magn. Reson. Imaging $3671-5$

Vonken E P, van Osch M J, Bakker C J and Viergever M 2000 A Simultaneous quantitative cerebral perfusion and Gd-DTPA extravasation measurement with dual-echo dynamic susceptibility contrast MRI Magn. Reson. Med. 43 820-7

Zaitsev M, Zilles K and Shah N J 2001 Shared $k$-space echo planar imaging with keyhole Magn. Reson. Med. 45 $109-17$ 